\title{
An Extensive Review on the Use of Feed Additives Against Fish Diseases and Improvement of Health Status of Fish in Turkish Aquaculture Sector
}

\author{
Sevdan Yılmaz ${ }^{1, *}\left(\mathbb{D}\right.$, Sebahattin Ergün ${ }^{1}\left(\mathbb{D}\right.$, Murat Yiğit $^{2}{ }^{(0)}$, Ebru Yılmaz $^{3} \oplus$ \\ ${ }^{1}$ Çanakkale Onsekiz Mart University, Faculty of Marine Sciences and Technology, Department of Aquaculture, Çanakkale, Turkey. \\ ${ }^{2}$ Çanakkale Onsekiz Mart University, Faculty of Marine Sciences and Technology, Department of Marine Technology Engineering, \\ Çanakkale, Turkey. \\ ${ }^{3}$ Aydın Adnan Menderes University, Faculty of Aqriculture, Department of Aquaculture, Aydin, Turkey.
}

\section{How to cite}

Yılmaz, S., Ergün, S., Yiğit, M., Yılmaz, E. (2022). An Extensive Review on the Use of Feed Additives Against Fish Diseases and Improvement of Health Status of Fish in Turkish Aquaculture Sector. Aquaculture Studies, 22(3), AQUAST710. http://doi.org/10.4194/AQUAST710

\section{Article History}

Received 09 July 2021

Accepted 27 December 2021

First Online 07 January 2022

\section{Corresponding Author}

Tel.: +0902862180018

E-mail: sevdanyilmaz@comu.edu.tr

\section{Keywords}

Aquaculture nutrition

Herbal additives

Fish health

Fish diseases

\begin{abstract}
Aquaculture is the second-fastest-growing sector in the world after informatics and its. Average growth of aquaculture is annually $8.8 \%$ over the last 30 years. Turkey has great potential in terms of fish production and the number of fish farms started to increase rapidly. Fish production in intensive culture conditions has enlarged possible threats of contagious disease outbreaks due to high stocking densities, water quality or environmental gradient, etc., as well as the combination of all these factors together. Depending on animal husbandry situations and organizational conditions, gradation of the aquatic surroundings and outbreaks of bacteriological diseases may well cause production losses around $30-40 \%$ in aquaculture facilities. Some fish diseases reported most repeatedly in Turkish aquaculture facilities are Vibriosis, Furunculosis, Streptococcosis, Lactococcosis, Aeromonas septicemia, Yersiniosis, Photobacteriosis and Flavobacteriosis. Antibiotics, disinfectants and chemotherapeutics used for the prevention and treatment of diseases result in residual antibiotics and chemicals in fish products, microorganisms resistant to antibiotics and damages to the aquatic environment and human health. This situation has led researchers to use alternative feed additives in fish diets such as medicinal plant, herbal extracts, phytochemicals, plant secondary metabolites, immunostimulants and probiotics. This review includes research conducted in Turkey between the years 2001 and 2020, and aims to summarize the findings regarding the use of medicinal plant, herbal extracts, phytochemicals, plant secondary metabolites and immunostimulants in fish feed to prevent and treat diseases, improve immunity, increase disease resistance, and reduce stress in fish towards a better management and best aquaculture practice for the sustainability of the growing aquaculture industry in the region and worldwide.
\end{abstract}

\section{Introduction}

Aquaculture represents one of the most commercially traded products in the world food industry, showing an annual increase of nearly $\sim 8.8 \%$ over the last 30 years (FAO, 2018). In Turkey, rainbow trout (Oncorhynchus mykiss) is the most produced species in inland waters, whereas European sea bass (Dicentrarchus labrax) and gilthead sea bream (Sparus aurata) production stands out in the marine environment. As of 2019, trout harvest from freshwater and marine facilities yielded 116.053 and 9.692 tons, respectively, whereas sea bream and sea bass harvest from marine cage farms in Turkey reached around 99.730 and 137.419 tons, respectively (TUIK, 2019).

Fish production in intensive culture conditions has enlarged possible threats of contagious disease outbreaks due to high stocking densities, water quality or environmental gradient, underwater acoustic noise beyond ambient audio level from machines in recirculating systems, etc., as well as the combination of all these factors together. Depending on animal husbandry situations and organizational conditions, gradation of the aquatic surroundings and outbreaks of 
bacteriological diseases may well cause production losses around $30-40 \%$ in aquaculture facilities. Some fish diseases reported most repeatedly in Turkish aquaculture facilities are Vibriosis, Furunculosis, Aeromonas septicemia, Streptococcosis, Lactococcosis, Yersiniosis, Photobacteriosis and Flavobacteriosis.

Antibiotics and chemotherapeutics are frequently chosen for the control of several infectious diseases in culture systems (Ozturk \& Altinok, 2014). There are 41 licenced drugs used in fish farm facilities in Turkey (Yarsan, 2013, Aksit, 2016). Out of these drugs, 15 contain florfenicol, 9 sulfadiazina+trimetoprim, 12 oxytetracycline, 2 enrofloxacin, 2 amoxicillin and 1 oxolinic acid (Yarsan, 2013).

The use of antibiotics and chemotherapeutics in aquaculture facilities is associated with residual complications in the nearby vicinity and the human body, as well as antibiotic-resistant pathogenic strains (Capkin et al., 2017; Yilmaz et al., 2018a). Additionally, resistance may be acquired by genes, which are placed on transportable elements of environmental, human, or other animals (pets)-origin of existing microorganisms that could be transported to the bacteria isolated from fish (Capkin et al., 2015).

Above mentioned detrimental effects have caused global concern in terms of the needs for biologically-safe feeding strategies in fish culture. Hence, fish diets used in aquaculture facilities could be improved with dietary incorporation of immunostimulants, organic acids, herbal extracts, medicinal plant, phytochemicals, plant secondary metabolites, essential oils and probiotics as feed additives. Many reports have documented the effect of $\beta$-glucans (Dugenci \& Candan, 2001; Sahan \& Duman, 2010), levamisole (Ispir \& Dorucu, 2005), medicinal plants (Dugenci et al., 2003; Gultepe et al., 2014; Diler et al., 2017a; Akyüz et al., 2018; Almabrok et al., 2018; Amhamed et al., 2018; Diler and Gormez 2019; Yilmaz and Er 2019; Bilen et al., 2020a; Öntaş et al., 2020), plant syrups (Yilmaz et al., 2018b, Yilmaz, 2019c; Yilmaz, 2020), spices (Yilmaz et al., 2013a; Yilmaz et al., 2013c; Yilmaz \& Ergun, 2014; Yilmaz et al., 2015a; Yilmaz et al., 2016; Gullu et al., 2016; Altunoglu et al., 2017; Savaşer et al., 2019), natural compounds (Yilmaz et al., 2015b; Acar, 2018a), organic acid (Yilmaz et al., 2019a), mushrooms (Ulukoy et al., 2016), yeast autolysates (Guven and Yalcın 2017), essential and plant oils (Acar et al., 2015; Arslan et al., 2018; Acar, 2018b; Altınterim et al., 2018; Altınterim \& Aksu 2019; Acar et al., 2019; Baba et al., 2016b; Kıvrak \& Didinen 2017; Diler et al., 2017b; Diler et al., 2017c; Yilmaz, 2019b; Kesbic, 2019a; Kesbic, 2019b; Parrino et al., 2019; Gultepe, 2020; Kesbic et al., 2020b), and anthocyanin (Yilmaz 2019d) as immune stimulators and health improvers in fish species (Table 1).

This study focuses mainly on the effects of different feed additives on fish, with special reference to hematology, serum biochemistry, nonspecific immunity, antioxidant status, stress responses, disease resistance and immune related gene expression. The results of this review are, therefore, aimed to provide remarkable information for the preparation of a "Standard Feed Additive Inoculation Protocol" and focus on several research gaps in fish production that might help to improve feeding strategies towards "Best Aquaculture Practice" for the sustainability of the growing aquaculture industry in Turkey as well as all around the world, both at laboratory and field levels.

\section{Aeromonas spp.}

Aeromonas hydrophila and Aeromonas veronii are among the bacteria that cause motile Aeromonas septicemia in fish (Austin \& Austin, 2016). Camellia sinensis seed powder (Er \& Kayıs, 2015), Capparis spinosa methanolic extract (Bilen et al., 2016a), Avena sativa water extract (Baba et al., 2016a), Zingiber officinale powder (Sahan et al., 2016), Anethum graveolens and Lepidium sativum methanolic extracts (Bilen et al., 2018), Malva sylvestris methanolic extract (Bilen et al., 2020b) and Pleurotus ostreatus methanolic extract (Bilen et al., 2016b) were administered to fish diets and found to increase protection against $A$. hydrophila (Table 2). Considering these earlier reports in terms of ecological approach (non-metanolic extraction) and the best RPS values, Avena sativa water extract (Baba et al., 2016a) with a value of 67 RPS and Camellia sinensis seed powder (Er \& Kayıs, 2015) with a value of 100 RPS are remarkable ones.

There was merely one report showing $5 \mathrm{~g} / \mathrm{kg}$ of caffeic acid supplement diets, adequately improved the enhanced nonspecific immune responses, up-regulated the immune and antioxidant related genes and disease resistance of fish against $A$. veronii (with a value of 57.89 RPS) in Turkey (Yilmaz, 2019a).

Yilmaz et al. (2020a) reported that L-alliin and oleuropein improved serum biochemistry parameters and immunological parameters of rainbow trout. Similary, Yilmaz et al. (2020c) investigated the nonspecific immune responses, health status and effect on growth performance of fish by testing black mulberry syrup in tilapia. The authors confirmed the highest RPS value $(68.75 \%)$ in the fish fed with black mulberry syrup by $2 \%$.

Aeromonas salmonicida are known as the causative agent of furunculosis. Yonar et al. (2019) reported that the curcumin powder administration, especially at $2 \%$ and $4 \%$, effectively enhanced the nonspecific immune responses and disease resistance to A. salmonicida subspecies achromogenes in rainbow trout, and the highest RPS value (76.67\%) was determined in the group fed with curcumin powder feed contribution by $2 \%$.

\section{Streptococcus spp.}

Streptococcal disease is caused by three major species of facultative anaerobic encapsulated Grampositive streptococci. Streptococcus iniae, S. agalactiae 
and S. dysgalactiae are in charge of disease in more than 30 freshwater, estuarine and marine fish species worldwide (Lee, 2015). Streptococcus iniae is perhaps the most significant because of its virulence across a broad range of hosts, global distribution, economic impact and zoonotic risk (Agnew et al., 2007; Gauthier, 2015).

Yilmaz et al. (2013b) indicated that rosemary, thyme or fenugreek powders increased disease resistance against Streptococcus iniae in tilapia fry (Table 3).

In another study, cumin can be used as a feed additive as an immunostimulant during feeding of Mozambique tilapia (Oreochromis mossambicus) and it can be recommended as an alternative to antibiotics to control streptococcal disease in tilapia culture (Yilmaz et al., 2012; Yilmaz et al., 2013c).

A different study revealed that the management of $400 \mathrm{mg} / \mathrm{kg}$ Tribulus terrestris extract, which are rich in flavonoids in a diet for Mozambique tilapia fry for 45 days and they improved disease resistance against $S$. iniae (Yilmaz et al., 2014).

Similarly, Yilmaz and Ergun (2014) stated that pimenta can be used as a substitute to antibiotics in the control of streptococcal disease in tilapia culture. The highest survival rate (80\%) was recorded in $10 \mathrm{~g} / \mathrm{kg}$ pimenta powder fed fish, and the lowest survival rate (38\%) was recorded in fish fed the control feed. Acar et al. (2015) reported that the highest RPS value (51.92) was found in the fish fed $1 \%$ sweet orange peel (Citrus sinensis). In addition, a plenty of studies have been reported on the use of feed additive against Streptococcus spp. infection (Table 3).

\section{Vibrio anguillarium}

Disases that are caused by Vibrio spp. are known to influence a wide range of wild and farmed fish species around the world, with reports in marine and brackish water environments, as well as in freshwater conditions (Woo \& Bruno, 2017; Gudding et al., 2014). V. anguillarium affects more than 50 diverse saltwater and freshwater fish species worldwide (Frans et al., 2011). Increased disease resistance against $V$. anguillarium was found in rainbow trout, gilthead seabream and European seabass when fed diets supplemented with Vaccinium myrtillus, Glycyrrhize glabra, Echinacea angustifolia (Terzioglu \& Diler, 2016), Salvia officinalis, Origanum vulgare (Diler et al., 2017c), and Artemisia vulgaris (Diler et al., 2018a), respectively (Table 4). Among these studies, the best RPS value with $66.67 \%$ at the end of experimental infection with $V$. anguillarum was detected in fish fed diets containing Cotinus coggygria leaf powder (Bilen et al., 2013).

\section{Yersinia ruckeri}

Infection with Yersinia ruckeri, a member of the family Enterobacteriaceae causes Yersiniosis in fish. The term "yersiniosis" is identical with enteric redmouth disease (ERM), a disease of fish caused by overt infection with Y. ruckeri.

ERM was first reported in the late 1950s when it was affecting rainbow trout in the Hagerman Valley of Idaho (Woo \& Bruno, 2017). Yersiniosis is now endemic across all the major salmonid farming areas of the world (Horne \& Barnes, 1999).

Gulec et. al., (2013) reported that dietary supplementation with thyme and fennel oils increased disease resistance in rainbow trout against $Y$. ruckeri (Table 5).

The oral administration of trans-cinnamic acid for 60 days in rainbow trout diet enhanced the haematological, serum biochemical and non-specific immune responses, improved immune-related gene expression as well as increased disease resistance (with a value of 50 RPS) against $Y$. ruckeri (Yilmaz \& Ergun, 2018).

Similarly, the highest RPS value $(42.09 \%)$ was detected in the group with $20 \%$ rosehip (Rosa canina) added (Sahan et al., 2017). It was observed that olive leaf ethanolic extract (Baba et al., 2018), pomegranate seed oil (Acar et al., 2018) and orange peel (Gultepe, 2020) reduced mortality in rainbow trout after challenge with Y. ruckeri (Table 5).

\section{Lactococcus garvieae}

In 1985, the genus Lactococcus was reported as a separate genus from Streptococcus. The original isolation of the L. garvieae was made from bovine mastitis (Schleifer et al., 1985; Teixeira et al., 1996; Collins et al., 1983).

The disease was described as a hyperacute systemic disease in rainbow trout (Eldar \& Ghittino, 1999). It can be separated into 3-distinct groups, based on RAPD analysis: Spanish, Portuguese, English and Turkish isolates formed one group, French and Italian formed a second, whilst Japanese formed a distinct third group (Ravelo et al., 2003).

Baba et al. (2017) examined the effects of argan oil, achieved from Argania spinosa, on pre- and postchallenge immuno-haematological and biochemical responses of the Nile tilapia (Oreochromis niloticus). For this purpose, the fish were comprising fed diets of 0 , $0.5 \%, 1 \%$ or $2 \%$ argan oil for 45 days and then, fish were challenged with L. garvieae. The highest RPS of $52.0 \%$ against $L$. garvieae were recorded in the fish fed on argan oil at $1 \%$ (Baba et al., 2017).

In another study with rainbow trout, the effects of the addition of olive pomace oil to assess the antioxidant activity and disease resistance were investigated (Yilmaz et al., 2020b). It was determined that the group fed with on $4 \%$ olive pomace oil had the highest RPS (43.86\%) (Yilmaz et al., 2020b). Moreover, rainbow trout were fed the different doeses of $O$. onites essential oil $(0.125,1.5,2.5$ and $3.0 \mathrm{~mL} / \mathrm{kg})$ for 90 days, and $3.0 \mathrm{~mL} / \mathrm{kg}$ diet showed no mortality after challenged 
with L. garvieae (Diler et al., 2017b).

Similarly, RPS values $55 \%$ was reported in study against L. garvieae in rainbow trout fed $2 \%$ supplemented Lentinula edodes (medicinal mushroom) water extract (Baba et al., 2015) (Table 6).

\section{Edwardsiella tarda}

The Edwardsiella (family Enterobacteriacae) was initially recognized as a new genus of the Enterobacteriaceae in the mid-1960s, and characterized 37 isolates recovered from open wounds, blood, urine and faeces of humans and animals (Ewing et al., 1965).

E. tarda has mainly been considered a warmwater, opportunistic fish pathogen. Environmental variables such as high temperature, poor water quality and high organic content contribute to the harshness of infections (Woo \& Capriano, 2017). E. tarda has also been reported from intensively reared rainbow trout in the Czech Republic (Rehulka et al., 2012). Diseased fish were anorexic and lethargic, having internal hyperaemia and petechial haemorrhages on the liver, with chronic lymphocytic portal hepatitis, focal necrosis, steatosis, dilation of the blood sinuses and activation of sinusoidal cells (Woo \& Cipriano, 2017).

Baba et al. (2016b) investigated the effects of citrus lemon peel essential oil on the antioxidant activity and disease resistance (E. tarda) of tilapia. The highest RPS value $(54.20 \%)$ was detected in the group with $0.5 \%$ citrus lemon peel essential oil added.

In another study, the effects of the addition of olive leaf ethanolic extract on $C$. carpio feeds on some blood parameters of fish, immune related genes and disease resistance (E. tarda) were investigated (Zemheri-Navruz et al., 2019). It was determined that the group with $1 \%$ olive leaf extract added had the highest RPS (43.75\%). Similarly, the highest survival rate of carp infected with E. tarda was found in groups with dill $(1 \mathrm{~g} / \mathrm{kg})$ and garden cress ( $2 \mathrm{~g} / \mathrm{kg}$ ) feed additive (Bilen et al., 2018).

\section{Plesiomonas shigelloides, Mycobacterium salmoniphilum and Spironucleus salmonis}

Blackberry syrup (Yilmaz 2019c), Rosa canina powder (Duman \& Sahan, 2018) and Artemisia campestris ethanolic extract (Diler et al., 2018b) were incorporated to fish diets and identified to increase protection against Plesiomonas shigelloides, Mycobacterium salmoniphilum and Spironucleus salmonis, respectively (Table 8 ). Duman and Sahan (2018) found the highest RPS value as $47.61 \%$ in the group with $15 \%$ Rosa canina powder addition. Diler et al. (2018b) determined the highest RPS value as $87.50 \%$ in the $1 \mathrm{~g} / \mathrm{kg} \mathrm{A}$. campestris (L) ethanol extract incorporation group, which determined the in vivo antiparasitic activity of Artemisia campestris (L) plant ethanol extract on Spironucleosis (Hexamitiasis) infections seen in rainbow trout.

\section{Conclusion and Perspectives}

Until recent years, antibiotics have been used as a feed additive that encouraged development in fish feed quality, and the use of antibiotics as a substance that promotes development has been banned in EU countries including Turkey. Many products considered as alternatives to antibiotics have been investigated. Result from intensive research challenges provide strong and reliable evidences that medicinal plants, herbal extracts, phytochemicals, plant secondary metabolites, immunostimulants, probiotics etc., are available in place of antibiotics in the fish diets. The positive effects of these additives especially killing pathogenic microorganisms stands in their strong ability of developing in the digestive organs, inhibiting the development of toxins in feed, increasing the activity of digestive enzymes, strengthening the immune system and, as a result, improving the growth performance and disease resistance of fish.

The exponential increase of the aquaculture industry is undeniable. As a result of the growing pressure from intensive production, environmental load from biogenic wastes such as organic waste and inorganic nutrients is likely to increase remarkably in the near future. Hence, using alternative feed additives with higher nutrient utilization efficiency might help to reduce waste load into the environment that in terms may support best aquaculture practice in long run.

As a result of this review, important information has been presented regarding the use of medicinal and aromatic plants as fish feed additives. Fish feed producers may encounter difficulties in accessing to medicinal or aromatic plants. In Turkey, there is a wide variety of medicinal and aromatic plants, a good source of feed additives with significantly strong antimicrobial and antioxidant effects. Approximately $6 \%$ of the world's medicinal and aromatic plants are naturally grown in Turkey and the production of 72 different kinds of medicinal and aromatic plants such as thyme, rosemary, garlic, sumac, ginger, turmeric, mint, etc is supported by the Turkish Agriculture and Rural Development Support Institution.

In the light of this information, feed companies are encouraged to benefit from medicinal and aromatic plants, which are abundant in Turkey, easily accessible at affordable prices, and whose effectiveness on fish health and welfare has been proven by intensive scientific studies.

\section{Ethical Statement}

No ethical statement required.

\section{Funding Information}

No funding required. 
Table 1. The role of beneficial feed ingredients supplemented to fish feed in Turkey at laboratory and field levels

\begin{tabular}{|c|c|c|c|c|c|c|}
\hline Additives & Fish species & $\begin{array}{c}\text { Initial body } \\
\text { weight }\end{array}$ & Stocking density $\left(\mathrm{kg} / \mathrm{m}^{3}\right)$ & $\begin{array}{c}\text { Doses and supplementation } \\
\text { duration }\end{array}$ & Results & References \\
\hline \multicolumn{7}{|l|}{ Extracts, powders, organic acids } \\
\hline $\begin{array}{l}\text { Viscum album, Urtica dioica and Zingiber } \\
\text { officinale (aqueous extract) }\end{array}$ & O. mykiss & $41 \mathrm{~g}$ & 0.82 & 0.1 and $1 \%, 3$ weeks & NSIRs $\uparrow$ & Dugenci et al. (2003) \\
\hline \multirow{2}{*}{$\begin{array}{l}\text { Thymus vulgaris, Rosmarinus officinalis and } \\
\text { Trigonella foenum graecum L. (powders) }\end{array}$} & D. labrax & $20.43 \pm 0.03 \mathrm{~g}$ & 2.48 & $1 \%, 45$ days & HS 个 & Yilmaz et al. (2013a) \\
\hline & D. labrax & $20.4 \pm 0.1 \mathrm{~g}$ & 2.47 & $1 \%, 45$ days & NSIRs $\uparrow$ and $\mathrm{HS} \uparrow$ & Yilmaz et al. (2016) \\
\hline Tribulus terrestris (extract) & O. niloticus & $2.61 \pm 0.35 \mathrm{~g}$ & 6.26 & $200,400,600 \mathrm{mg} / \mathrm{kg}, 88$ days & $\mathrm{HS} \uparrow$ and NSIRs $\uparrow$ & Gultepe et al. (2014) \\
\hline \multirow{2}{*}{ Pimenta dioica (powder) } & O. mossambicus & $20.05 \pm 0.10 \mathrm{~g}$ & 4.51 & $5,10,15,20 \mathrm{~g} / \mathrm{kg}, 60$ days & NSIRs $\uparrow, H S \uparrow$ and ASS $\downarrow$ & Yilmaz et al. (2015a) \\
\hline & O. mossambicus & $16.05 \pm 0.08 \mathrm{~g}$ & 3.61 & $5,10,15,20 \mathrm{~g} / \mathrm{kg}, 60$ days & $\mathrm{HS} \uparrow$ and NSIRs $\uparrow$ & Gullu et al. (2016) \\
\hline Carvacrol & O. mykiss & $10.79 \pm 0.57$ & 0.44 & $1,3,5 \mathrm{~g} / \mathrm{kg}, 60$ days & HS $\uparrow$ and NSIRs $\uparrow$ & Yilmaz et al. (2015b) \\
\hline $\begin{array}{l}\text { Artemisia vulgaris } \\
\text { (powder and ethanolic extract) }\end{array}$ & O. mykiss & $21 \mathrm{~g}$ & 1.10 & $\begin{array}{c}\text { Powder: } 0.1,0.5,1,2 \% \\
\text { Extract: } 250,1000 \mathrm{mg} / \mathrm{kg}, 90 \text { days }\end{array}$ & AS $\uparrow$ & Diler et al. (2017a) \\
\hline Black cumin (methanolic extract) & O. mykiss & $15.02 \pm 0.01 \mathrm{~g}$ & 1.00 & 0.1 and $0.5 \mathrm{~g} / \mathrm{kg}, 30$ days & NSIRs $\uparrow$ & Altunoglu et al. (2017) \\
\hline Tilia tomentosa (methanolic extract) & C. carpio & $4.35 \pm 0.16 \mathrm{~g}$ & no information available & $0.01,0.05,0.1 \%, 45$ days & NSIRs $\uparrow$ & Almabrok et al. (2018) \\
\hline Chenopodium album (methanolic extract) & C. carpio & $2.4 \pm 0.1 \mathrm{~g}$ & 0.87 & $0.5,0.1,1 \mathrm{~g} / \mathrm{kg}, 45$ days & NSIRs $\uparrow$ & Amhamed et al. (2018) \\
\hline Propolis (ethanolic extract) & O. mossambicus & $15.43 \pm 0.16 \mathrm{~g}$ & 6.17 & 2 and $4 \mathrm{~g} / \mathrm{kg}, 60$ days & NSIRs $\uparrow$ & Acar (2018a) \\
\hline $\begin{array}{l}\text { Cherry laurel (Laurocerasus officinalis } \\
\text { Roem.) leaf extract }\end{array}$ & O. mykiss & $6.05 \pm 0.03 \mathrm{~g}$ & 0.045 & $1,5,10,15 \mathrm{~g} / \mathrm{kg}, 52$ days & $\mathrm{SR} \uparrow, \mathrm{AS} \uparrow$ & Akyüz et al. (2018) \\
\hline root powder & O. mykiss & $108.7 \pm 17.0 \mathrm{~g}$ & no information available & $0.0,0.5,1.0,2.5,5.0,10.0$ and $20.0 \mathrm{~g} / \mathrm{kg}$ & HS 个, NSIRs $\uparrow$ & Savaşer et al. (2019) \\
\hline Cinnamic acid & O. mykiss & $17.49 \pm 0.08 \mathrm{~g}$ & 3.74 & $250,500,750,1500 \mathrm{mg} / \mathrm{kg}, 60$ days & $\mathrm{AGER} \uparrow, \mathrm{AS} \uparrow, \mathrm{HS} \uparrow$ & Yilmaz et al. (2019a) \\
\hline $\begin{array}{l}\text { Artemisia campestris and A. absinthium } \\
\text { (ethanolic extracts) }\end{array}$ & O. mykiss & $1.5-2 \mathrm{~g}$ & 0.86 & $1,2,3 \mathrm{~g} / \mathrm{kg}, 21$ days & $\operatorname{IM} \uparrow$ & Diler \& Gormez (2019) \\
\hline Fig and rosemary extract & O. mykiss & $12.47 \pm 0.15$ & 0.34 & $0.5,1,2 \mathrm{~g} / \mathrm{kg}, 60$ days & $\mathrm{HS} \uparrow$ & Yilmaz \& Er (2019) \\
\hline Melissa officinalis (extract) & O. mykiss & $12.04 \pm 0.71 \mathrm{~g}$ & 0.35 & $0.1,0.5,1 \mathrm{~g} / \mathrm{kg}, 75$ days & HS $\uparrow, N S I R s \uparrow$ & Bilen et al. (2020a) \\
\hline $\begin{array}{l}\text { Crocus cancellatus subsp. mazziaricus } \\
\text { (Herbert) Mathew extract }\end{array}$ & D. labrax & $130 \pm 3.5 \mathrm{gr}$ & No information available & $0.5 \mathrm{mg} /$ fish, $2 \mathrm{mg} / \mathrm{fish}$ & NSIRs $\uparrow$ & Öntaş et al. (2020) \\
\hline \multicolumn{7}{|l|}{ Fruits and Plants Syrups } \\
\hline Carob syrup & O. mossambicus & $30.70 \pm 1.58 \mathrm{~g}$ & 6.57 & $0.625,1.25,2.5,5 \%, 60$ days & HS $\uparrow$ and NSIRs $\uparrow$ & Yilmaz et al. (2018b) \\
\hline Carob syrup & O. niloticus & $3.68 \pm 0.092 \mathrm{~g}$ & 0.78 & $0.625,1.25,2.5,5 \%, 60$ days & $\begin{array}{c}\text { NSIRs } \uparrow, H S \uparrow, \\
\text { IRGER } \uparrow, A G E R ~ \uparrow \text { and AMS } \uparrow\end{array}$ & Yilmaz (2020) \\
\hline \multicolumn{7}{|l|}{ Fruit pigments } \\
\hline Anthocyanin & O. niloticus & $8.24 \pm 0.64 \mathrm{~g}$ & 1.76 & $20,40,80,160 \mathrm{mg} / \mathrm{kg}, 60$ days & $\begin{array}{l}\text { IRGER } \uparrow, A G E R \uparrow, A S \uparrow, \\
\text { NSIRs } \uparrow \text { and AMS } \downarrow\end{array}$ & Yilmaz (2019d) \\
\hline \multicolumn{7}{|l|}{ Essential oils and plant oils } \\
\hline Rosmarinus officinalis & O. mykiss & $10.14 \pm 0.06 \mathrm{~g}$ & 0.72 & $0.025,0.05$ and $0.1 \%, 60$ days & NSIRs $\uparrow$ & Kıvrak \& Didinen (2017) \\
\hline Lavandula stoechas (oil) & C. carpio & $10.88 \pm 0.90 \mathrm{~g}$ & 2.33 & 5 and $10 \mathrm{~g} / \mathrm{kg}, 60$ days & $\mathrm{HS} \uparrow$ & Yilmaz (2019b) \\
\hline Juniper berry (oil) & C. carpio & $3.07 \pm 0.15 \mathrm{~g}$ & 2.30 & 5 and $10 \mathrm{~mL} / \mathrm{kg}, 60$ days & $\mathrm{HS} \uparrow$ & Kesbic (2019a) \\
\hline Cinnamon (oil) & O. mykiss & $10.68 \pm 0.35 \mathrm{~g}$ & 1.60 & $1,2,4,10 \mathrm{~mL} / \mathrm{kg}, 60$ days & $\mathrm{HS} \uparrow$ & Kesbic (2019b) \\
\hline Hot pepper (oil) & O. mykiss & $\sim 7 \mathrm{~g}$ & no information available & $1,2,4,6 \%, 60$ days & $\mathrm{HS} \uparrow$ & Parrino et al. (2019) \\
\hline Hypericum perforatum (oil) & C. carpio & $3.07 \pm 0.02 \mathrm{~g}$ & 2.30 & 5 and $10 \mathrm{~g} / \mathrm{kg}, 60$ days & $\mathrm{HS} \uparrow$ & Acar (2018b) \\
\hline Vitis vinifera seed (oil) & O. mykiss (fry) & $30 \mathrm{~g}$ & 1.2 & $250,500,1000 \mathrm{mg} / \mathrm{kg}, 60$ days & $\mathrm{HS} \uparrow, \mathrm{AS} \uparrow$ and $\mathrm{SR} \uparrow$ & Arslan et al. (2018) \\
\hline Green tea (Camellia sinensis) oil & O. mykiss & $76.25 \pm 2.75 \mathrm{~g}$ & no information available & $0.25,0.5,1.0 \%, 42$ days & NSIRs $\uparrow$ & Altınterim al.(2018) \\
\hline $\begin{array}{l}\text { Allium sativum Limne, } \\
\text { Allium tuncelianum Kollman oils }\end{array}$ & O. mykiss & $39.0 \pm 1.64 \mathrm{~g}$ & 9.75 & $2 \%$ & NSIRs $\uparrow, N B T \downarrow$ & Altınterim \& Aksu (2019) \\
\hline
\end{tabular}




\begin{tabular}{|c|c|c|c|c|c|c|}
\hline Additives & Fish species & $\begin{array}{l}\text { Initial body } \\
\text { weight }\end{array}$ & Stocking density $\left(\mathrm{kg} / \mathrm{m}^{3}\right)$ & $\begin{array}{l}\text { Doses and supplementation } \\
\text { duration }\end{array}$ & Results & References \\
\hline \multirow{2}{*}{ Bergamot peel oil } & D. labrax & $5.10 \pm 0.05 \mathrm{~g}$ & 2.04 & $0.5,1,2 \%, 60$ days & NSIRs $\uparrow$ & Acar et al. (2019) \\
\hline & O.niloticus & $2.57 \pm 0.06 \mathrm{~g}$ & 2.45 & $0.5,1,2 \%, 8$ weeks & NSIRs $\uparrow$ and HS $\uparrow$ & Kesbic et al. (2020a) \\
\hline Monterey cypress Leaf essential oil & C.carpio & $7.86 \pm 0.15 \mathrm{~g}$ & 1.96 & $0.5,0.75,1 \%, 60$ days & HS $\uparrow$ & Kesbic et al. (2020b) \\
\hline \multicolumn{7}{|l|}{ Mushrooms } \\
\hline Lentinula edodes (water extract) & O. mykiss & $56.2 \pm 6.6 \mathrm{~g}$ & 11.94 & 1 and $2 \%, 6$ weeks & Reduce lipid in liver & Ulukoy et al. (2016) \\
\hline \multicolumn{7}{|l|}{ Yeast autolysates } \\
\hline Saccharomyces cerevisiae & O. mykiss & $35 \mathrm{~g}$ & 0.7 & 0.1 and $0.2 \%, 8$ weeks & NSIRs $\uparrow$ and HS $\uparrow$ & Guven \& Yalcın (2017) \\
\hline \multicolumn{7}{|l|}{$\beta$-glucans } \\
\hline MacroGard & O. mykiss & $54 \mathrm{~g}$ & 1.59 & 0.1 and $1 \%, 3$ weeks & NSIRs $\uparrow$ & Dugenci \& Candan (2001) \\
\hline$\beta-1,3 / 1,6$ glucan & O.niloticus & $25.61 \pm 0.03 \mathrm{~g}$ & 0.3 & 0.1 and $0.5 \%, 2$ weeks & NSIRs $\uparrow$ & Sahan \& Duman (2010) \\
\hline \multicolumn{7}{|l|}{ Levamisole } \\
\hline & O. mykiss & 99.4 and $216.0 \mathrm{~g}$ & 6.27 and 13.64 & $5 \mathrm{mg} / \mathrm{kg}$ fish (intraperitonally), 14 days & NSIRs $\uparrow$ & Ispir \& Dorucu (2005) \\
\hline
\end{tabular}

Table 2. Studies addressing beneficial uses of fish feed additives against Aeromonas spp.

\begin{tabular}{|c|c|c|c|c|c|c|}
\hline Additives & Fish species & $\begin{array}{l}\text { Initial body } \\
\text { weight }\end{array}$ & $\begin{array}{l}\text { Stocking } \\
\text { density } \\
\left(\mathrm{kg} / \mathrm{m}^{3}\right)\end{array}$ & $\begin{array}{c}\text { Doses and supplementation } \\
\text { duration }\end{array}$ & Results & References \\
\hline \multicolumn{7}{|l|}{ Extracts, powders, organic acids, compounds } \\
\hline Camellia sinensis (seed powder) & O. mykiss & $10 \pm 0.12 \mathrm{~g}$ & 4 & $10 \%, 10$ days & DR to A. hydrophila & Er and Kayıs (2015) \\
\hline Capparis spinosa (methanolic extract) & O. mykiss & $12.04 \pm 0.71 \mathrm{~g}$ & 0.80 & 0.1 and $0.5 \mathrm{~g} / \mathrm{kg}, 30$ days & IRGER $\uparrow$, NSIRs $\uparrow$ and DR to A. hydrophila & Bilen et al. (2016a) \\
\hline Avena sativa (water extract) & C. carpio & $9.91 \pm 1.52 \mathrm{~g}$ & 3.56 & $5,10,20 \mathrm{~g} / \mathrm{kg}, 60$ days & NSIRs $\uparrow$ and DR to A. hydrophila & Baba et al. (2016a) \\
\hline Pleurotus ostreatus (methanolic extract) & O. mykiss & $10.28 \pm 0.1 \mathrm{~g}$ & 1.03 & 0.1 and $0.5 \mathrm{~g} / \mathrm{kg}, 30$ days & NSIRs $\uparrow$ and DR to A. hydrophila & Bilen et al. (2016b) \\
\hline Zingiber officinale powder & O. niloticus & $25.61 \pm 0.03 \mathrm{~g}$ & 0.12 & $0.1,0.5,1 \%, 90$ days & 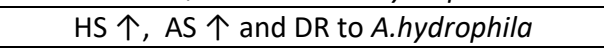 & Sahan et al. (2016) \\
\hline $\begin{array}{l}\text { Anethum graveolens and Lepidium sativum } \\
\text { (methanolic extract) }\end{array}$ & C. carpio & $3.46 \pm 0.1 \mathrm{~g}$ & 1.76 & 1 and $2 \mathrm{~g} / \mathrm{kg}$, 45 days & NSIRs $\uparrow$, and DR to $A$. hydrophila & Bilen et al. (2018) \\
\hline Caffeic acid & O. niloticus & $17.49 \pm 0.08 \mathrm{~g}$ & 3.74 & $1,5,10 \mathrm{~g} / \mathrm{kg}, 60$ days & $\begin{array}{c}\text { NSIRs } \uparrow, \text { IRGER } \uparrow, A G E R ~ \uparrow, A S \uparrow, H S \uparrow \text { and } \\
\text { DR to A. veronii }\end{array}$ & Yilmaz (2019a) \\
\hline Curcumin (powder) & O. mykiss & $31.29 \pm 1.17 \mathrm{~g}$ & 1.44 & $1,2,4 \%, 8$ weeks & $\begin{array}{c}\mathrm{HS} \uparrow, \mathrm{NSIRs} \uparrow, \mathrm{AS} \uparrow \text { and DR to Aeromonas } \\
\text { salmonicida subsp. achromogenes }\end{array}$ & Yonar et al. (2019) \\
\hline L-alliin and Oleuropein & O. mykiss & $12.6 \pm 0.91 \mathrm{~g}$ & 2.57 & $10 \mathrm{mg} / \mathrm{kg}, 60$ days & HS $\uparrow$ and DR to A.salmonicida & Yilmaz et al. (2020a) \\
\hline $\begin{array}{l}\text { Malva sylvestris } \\
\text { (Methanolic extract) }\end{array}$ & O. mykiss & $54.97 \pm 0.03 \mathrm{~g}$ & 3.66 & 0.1 and $0.5 \mathrm{~g} / \mathrm{kg}, 30$ days & NSIRs $\uparrow$, IRGER $\uparrow$, DR to A. hydrophila & Bilen et al. (2020b) \\
\hline \multicolumn{7}{|l|}{ Fruits and Plants Syrups } \\
\hline Black mulberry syrup & O. niloticus & $9.74 \pm 0.9 \mathrm{~g}$ & 2.08 & $0.75,1.5,2.0,3.0 \%, 60$ days & NSIRs $\uparrow, H S$ 个, IRGER $\uparrow$, and DR to A. veronii & Yilmaz et al. (2020c) \\
\hline
\end{tabular}

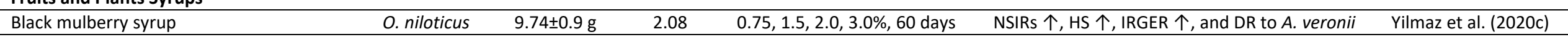

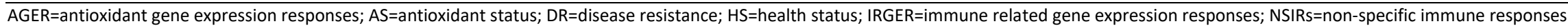


Table 3. Studies addressing beneficial uses of fish feed additives against Streptococcus spp.

\begin{tabular}{|c|c|c|c|c|c|c|}
\hline Additives & Fish species & $\begin{array}{l}\text { Initial body } \\
\text { weight }\end{array}$ & $\begin{array}{c}\text { Stocking } \\
\text { density } \\
\left(\mathrm{kg} / \mathrm{m}^{3}\right)\end{array}$ & $\begin{array}{c}\text { Doses and supplementation } \\
\text { duration }\end{array}$ & Results & References \\
\hline \multicolumn{7}{|l|}{ Extracts, powders } \\
\hline \multirow{2}{*}{ Cuminum cyminum } & O. mossambicus & $0.56 \pm 0.02 \mathrm{~g}$ & 0.015 & $0.5,1,1.5,2 \%, 75$ days & DR to $S$. iniae & Yilmaz et al. (2012) \\
\hline & O. mossambicus (fry) & $0.012 \pm 0.001 \mathrm{~g}$ & 0.02 & $0.5,1,1.5,2 \%, 45$ days & DR to S. iniae & Yilmaz et al. (2013c) \\
\hline $\begin{array}{l}\text { Thymus vulgaris, Rosmarinus officinalis and } \\
\text { Trigonella foenum graecum L. (powders) }\end{array}$ & O. mossambicus (fry) & $0.012 \pm 0.001 \mathrm{~g}$ & 0.02 & $1 \%, 45$ days & DR to S. iniae & Yilmaz et al. (2013b) \\
\hline Tribulus terrestris (extract) & O. mossambicus (fry) & $0.0120 \mathrm{~g}$ & 0.02 & $200,400,600,500 \mathrm{mg} / \mathrm{kg}$, 45 days & PRHD $\downarrow$ and DR to S. iniae & Yilmaz et al. (2014) \\
\hline Pimenta dioica (powder) & O. mossambicus (fry) & $0.012 \mathrm{~g}$ & 0.02 & $5,10,15,20 \mathrm{~g} / \mathrm{kg}, 50$ days & DR to $S$. iniae & Yilmaz and Ergun (2014) \\
\hline \multicolumn{7}{|l|}{ Essential oils } \\
\hline Sweet orange peel & O. mossambicus & $0.91 \pm 0.03 \mathrm{~g}$ & 0.56 & $0.1,0.3,0.5 \%, 90$ days & NSIRs $\uparrow$ and DR to $S$. iniae & Acar et al. (2015) \\
\hline
\end{tabular}

$\mathrm{DR}=$ disease resistance; NSIRs=non-specific immune responses; $\mathrm{PRHD}=$ pathogen related histological damage

Table 4. Studies addressing beneficial uses of fish feed additives against Vibrio anguillarium

\begin{tabular}{|c|c|c|c|c|c|c|}
\hline Additives & Fish species & Initial body weight & $\begin{array}{c}\text { Stocking } \\
\text { density } \\
\left(\mathrm{kg} / \mathrm{m}^{3}\right)\end{array}$ & $\begin{array}{c}\text { Doses and supplementation } \\
\text { duration }\end{array}$ & Results & References \\
\hline \multicolumn{7}{|l|}{ Extracts, powders, essential oil } \\
\hline Cotinus coggygria leaf powder & C. carpio & $4.14 \pm 0.08 \mathrm{~g}$ & 1.81 & $\begin{array}{c}0.5,1,1.5 \mathrm{~g} / \mathrm{kg} \\
4 \text { weeks }\end{array}$ & $\begin{array}{c}\text { NSIRs } \uparrow \text { and DR to } V . \\
\text { anguillarum }\end{array}$ & Bilen et al. (2013) \\
\hline $\begin{array}{l}\text { Artemisia vulgaris } \\
\text { (powder and ethanolic extract) }\end{array}$ & O. mykiss & $\begin{array}{c}20.48 \pm 0.19 \mathrm{~g} \text { and } \\
20.81 \pm 0,04 \mathrm{~g}\end{array}$ & 0.35 and 0.36 & $\begin{array}{c}\text { Powder: } 0.1,0.5,1,2 \% \\
\text { Extract: } 250,1000 \mathrm{mg} / \mathrm{kg}, 45 \text { days }\end{array}$ & $\begin{array}{c}\text { NSIRs } \uparrow \text { and DR to } V . \\
\text { anguillarum }\end{array}$ & Diler et al. (2018a) \\
\hline $\begin{array}{l}\text { Vaccinium myrtillus, Glycyrrhize glabra, } \\
\text { Echinacea angustifolia and Salvia officinalis }\end{array}$ & O. mykiss & $15 \pm 2 \mathrm{~g}$ & 1.28 & 0.1 and $1 \%, 45$ days & $\begin{array}{c}\text { NSIRs } \uparrow \text { and DR to } V . \\
\text { anguillarum }\end{array}$ & $\begin{array}{l}\text { Terzioglu and Diler } \\
\text { (2016) }\end{array}$ \\
\hline $\begin{array}{l}\text { Cotinus coggygria and Malva sylvestris } \\
\text { (methanolic extract) }\end{array}$ & $\begin{array}{l}\text { S. aurata and } \\
\text { D. labrax }\end{array}$ & $\begin{array}{c}19.92 \pm 0.40 \mathrm{~g} \text { and } \\
18.66 \pm 0.86 \mathrm{~g}\end{array}$ & 1.10 and 1.03 & $\begin{array}{c}500,1000 \mathrm{mg} / \mathrm{kg} \\
60 \text { days }\end{array}$ & $\begin{array}{c}\text { NSIRs } \uparrow \text { and DR to } V . \\
\text { anguillarum }\end{array}$ & Bilen et al. (2019) \\
\hline Origanum vulgare L. essential oil & O. mykiss & $26 \mathrm{~g}$ & 0.45 & $0.125,1.5,2.5,3.0 \mathrm{ml} / \mathrm{kg}, 90$ days & DR to $V$. anguillarum & Diler et al. (2017c) \\
\hline
\end{tabular}

Origanum vulgare L. essential oil

DR to $V$. anguillarum 
Table 5. Studies addressing beneficial uses of fish feed additives against Yersinia ruckeri

\begin{tabular}{|c|c|c|c|c|c|c|}
\hline Additives & Fish species & $\begin{array}{l}\text { Initial body } \\
\text { weight }\end{array}$ & $\begin{array}{c}\text { Stocking } \\
\text { density } \\
\left(\mathrm{kg} / \mathrm{m}^{3}\right)\end{array}$ & $\begin{array}{c}\text { Doses and supplementation } \\
\text { duration }\end{array}$ & Results & References \\
\hline \multicolumn{7}{|l|}{ Extracts, powders, oils } \\
\hline Thyme and Fennel oils & O. mykiss & $84 \pm 1.02 \mathrm{~g}$ & 5.6 & $10 \mathrm{~mL} / 100 \mathrm{~g}, 1$ week & HS $\uparrow$ after $Y$. ruckeri infection & Gulec et al. (2013) \\
\hline Rosa canina powder & O. mykiss & 50 to $60 \mathrm{~g}$ & 2.5 to 3 & $10,20,30 \%, 50$ days & HS $\uparrow$, AS $\uparrow, ~ N S I R s ~ \uparrow$ and DR to Y.ruckeri & Sahan et al. (2017) \\
\hline Pomegranate seed (oil) & O. mykiss & $6.79 \pm 0.02 \mathrm{~g}$ & 2.71 & $0.5,1,2 \%, 60$ days & HS $\uparrow$ and DR to $Y$. ruckeri & Acar et al. (2018) \\
\hline Olive leaf (ethanolic extract) & O. mykiss & $51.22 \pm 3.04 \mathrm{~g}$ & 5.48 & $0.1,0.25,0.5,1 \%, 60$ days & IRGER $\uparrow$, NSIRs $\uparrow, H S \uparrow$ and DR to $Y$. ruckeri & Baba et al. (2018) \\
\hline Cinnamic acid & O. mykiss & $17.01 \pm 0.05 \mathrm{~g}$ & 3.64 & $250,500,750,1500 \mathrm{mg} / \mathrm{kg}, 60$ days & NSIRs $\uparrow$, IRGER $\uparrow, H S \uparrow$ and DR to $Y$. ruckeri & Yilmaz and Ergun (2018) \\
\hline Orange peel essential oil & O. mykiss & $4.48 \pm 0.03 \mathrm{~g}$ & 2.46 & $0.5,1,3 \mathrm{~mL} / \mathrm{kg}, 90$ days & NSIRs $\uparrow, H S \uparrow, D R$ to $Y$. ruckeri & Gultepe, (2020) \\
\hline
\end{tabular}

$\mathrm{AS}=$ =antioxidant status; $\mathrm{DR}=$ disease resistance; $\mathrm{HS}=$ health status; IRGER=immune related gene expression responses; $N S I R s=n o n$-specific immune responses

Table 6. Studies addressing beneficial uses of fish feed additives against Lactococcus garvieae

\begin{tabular}{|c|c|c|c|c|c|c|}
\hline Additives & Fish species & Initial body weight & $\begin{array}{c}\text { Stocking density } \\
\left(\mathrm{kg} / \mathrm{m}^{3}\right)\end{array}$ & $\begin{array}{c}\text { Doses and supplementation } \\
\text { duration }\end{array}$ & Results & References \\
\hline \multicolumn{7}{|l|}{ Plant oils, essential oils } \\
\hline Argan oil & O. mykiss & $18.31 \pm 0.10 \mathrm{~g}$ & 6.59 & $0.5,1,2 \%, 45$ days & NSIRs $\uparrow$ and DR to L. garvieae & Baba et al. (2017) \\
\hline Olive pomace oil & O. mykiss & $12.10 \pm 0.13 \mathrm{~g}$ & 2.59 & $4,8,12 \%, 60$ days & HS $\uparrow$ and DR to L. garvieae. & Yilmaz et al. (2020b) \\
\hline Origanum onites essential oil & O. mykiss & $26.05 \pm 0.15 \mathrm{~g}$ & 5.21 & $0.125,1.5,2.5,3 \mathrm{~mL} / \mathrm{kg}$, 90 days & DR to L. garvieae & Diler et al. (2017b) \\
\hline \multicolumn{7}{|l|}{ Mushrooms } \\
\hline Lentinula edodes (water extract) & O. mykiss & $20 \mathrm{~g}$ & 0.6 & 1 and $2 \%, 6$ weeks & NSIRs $\uparrow$ and DR to L. garvieae & Baba et al. (2015) \\
\hline
\end{tabular}


Table 7. Studies addressing beneficial uses of fish feed additives against Edwardsiella tarda

\begin{tabular}{|c|c|c|c|c|c|c|}
\hline Additives & Fish species & $\begin{array}{l}\text { Initial body } \\
\text { weight }\end{array}$ & $\begin{array}{c}\text { Stocking } \\
\text { density } \\
\left(\mathrm{kg} / \mathrm{m}^{3}\right)\end{array}$ & $\begin{array}{c}\text { Doses and supplementation } \\
\text { duration }\end{array}$ & Results & References \\
\hline \multicolumn{7}{|l|}{ Extracts } \\
\hline $\begin{array}{l}\text { Anethum graveolens and Lepidium } \\
\text { sativum (methanolic extract) }\end{array}$ & C. carpio & $3.46 \pm 0.1 \mathrm{~g}$ & 1.76 & 1 and $2 \mathrm{~g} / \mathrm{kg}$, 45 days & NSIRs $\uparrow$, and DR to $E$. tarda & Bilen et al. (2018) \\
\hline Olive leaf (ethanolic extract) & C. carpio & $15.90 \pm 0.93 \mathrm{~g}$ & 0.318 & $0.1,0.25,0.5,1 \%, 60$ days & $\begin{array}{c}\text { IRGER } \uparrow, \text { NSIRs } \uparrow, H S ~ \uparrow \text { and DR to } \\
\text { E. tarda }\end{array}$ & Zemheri-Navruz et al. (2019) \\
\hline \multicolumn{7}{|l|}{ Essential oils } \\
\hline Citrus limon peel & O. mossambicu & $12.87 \pm 0.18 \mathrm{~g}$ & 6.43 & $0.5,0.75,1 \%, 60$ days & NSIRs $\uparrow, ~ H S ~ \uparrow$ and DR to E. tarda & Baba et al. (2016b) \\
\hline
\end{tabular}

Citrus limon pee

O. mossambicus $12.87 \pm 0.18 \mathrm{~g} \quad 6.43$

NSIRs 个, HS 个 and DR to E. tarda

Baba et al. (2016b)

$\mathrm{DR}=$ disease resistance; $\mathrm{HS}=$ health status; IRGER=immune related gene expression responses; NSIRs=non-specific immune responses

Table 8. Studies addressing beneficial uses of fish feed additives against Plesiomonas shigelloides, Mycobacterium salmoniphilum and Spironucleus salmonis

\begin{tabular}{|c|c|c|c|c|c|c|}
\hline Additives & Fish species & $\begin{array}{l}\text { Initial body } \\
\text { weight }\end{array}$ & $\begin{array}{c}\text { Stocking } \\
\text { density } \\
\left(\mathrm{kg} / \mathrm{m}^{3}\right)\end{array}$ & $\begin{array}{c}\text { Doses and supplementation } \\
\text { duration }\end{array}$ & Results & References \\
\hline \multicolumn{7}{|l|}{ Powders, Extracts } \\
\hline Rosa canina powder & A.gueldenstaedtii & $307.8 \pm 11.4-\mathrm{g}$ & 1.53 & $5,10,15 \%, 35$ days & NSIRs $\uparrow$ and DR to M. salmoniphilum & Duman and Sahan (2018) \\
\hline $\begin{array}{l}\text { Artemisia campestris } \\
\text { (ethanolic extract) }\end{array}$ & O. mykiss & $1.5-2.0 \mathrm{~g}$ & 0.18 & $\begin{array}{c}1.0,1.5,2.0,2.5,3.0 \mathrm{~g} / \mathrm{kg}, \\
7 \text { and } 21 \text { days }\end{array}$ & DR to S. salmonis & Diler et al. (2018b) \\
\hline \multicolumn{7}{|c|}{ Fruits and Plants Syrups } \\
\hline Blackberry syrup & O. niloticus & $26.75 \pm 2.67 \mathrm{~g}$ & 5.73 & $\begin{array}{l}7.5,15,30 \mathrm{~g} / \mathrm{kg} \\
90 \text { days }\end{array}$ & 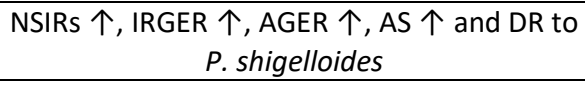 & Yilmaz (2019c) \\
\hline
\end{tabular}

AGER=antioxidant gene expression responses; AS=antioxidant status; DR=disease resistance; IRGER=immune related gene expression responses; NSIRs=non-specific immune responses 


\section{Author Contribution}

Authors shared equally in this work. All authors have read and agreed to the published version of the manuscript.

\section{Conflict of Interest}

The authors declare that they have no conflict interest.

\section{References}

Acar, U. (2018a). Effects of diet supplemented with ethanolic extract of propolis on growth performance, hematological and serum biochemical parameters and disease resistance of Mozambique tilapia (Oreochromis mossambicus) against Streptococcus iniae. Aquaculture, 495, 339-344.

https://doi.org/10.1016/j.aquaculture.2018.06.007

Acar, U. (2018b). Sarı kantaron (Hypericum perforatum) yağının sazan yavrularının (Cyprinus carpio) büyüme performansı ve bazı kan parametreleri üzerine etkisi. Alınteri Zirai Bilimler Dergisi, 33(1), 21-27. https://doi.org/10.28955/alinterizbd.343202

Acar, U., Kesbic, O. S., Inanan, B. E., \& Yilmaz, S. (2019). Effects of dietary Bergamot (Citrus bergamia) peel oil on growth, haematology and immune response of European sea bass (Dicentrarchus labrax) juveniles. Aquaculture Research, 50(11), 3305-3312. https://doi.org/10.1111/are.14288

Acar, U., Kesbic, O. S., Yilmaz, S., Gultepe, N., \& Turker, A. (2015). Evaluation of the effects of essential oil extracted from sweet orange peel (Citrus sinensis) on growth rate of tilapia (Oreochromis mossambicus) and possible disease resistance against Streptococcus iniae. Aquaculture, 437, 282-286.

https://doi.org/10.1016/j.aquaculture.2014.12.015

Acar, U., Parrino, V., Kesbic, O. S., Lo Paro, G., Saoca, C., Abbate, F., Yilmaz, S., \& Fazio, F. (2018). Effects of different levels of pomegranate seed oil on some blood parameters and disease resistance against Yersinia ruckeri in rainbow trout. Frontiers in Physiology, 9, 596. https://doi.org/10.3389/fphys.2018.00596 Altınterim, B. \& Aksu, Ö. (2019). Masere sarımsak (Allium sativum Limne) ve Tunceli sarımsağı (Allium tuncelianum Kollman) yağlarının yoğun stoklanmış gökkuşağı alabalıklarının (Oncorhynchus mykiss W.) bazı kan parametrelerine ve NBT (Nitroblue Tetrazolium) seviyelerine etkileri. BAUN Fen Bilimleri Enstitüsü Dergisi, 21(2): 716-723.

https://doi.org/10.25092/baunfbed.637083

Altınterim, B., Öztürk, E., Kutluyer, F., \& Aksu, Ö. (2018). Yeşil çay yağının gökkuşağı alabalıklarının (Oncorhynchus mykiss) yem değerlendirme oranına ve hematolojik parametrelerine etkileri. Atatürk Üniversitesi Veteriner Bilimleri Dergisi, 13(2): 159-164. https://doi.org/ 10.17094/ataunivbd.296989

Agnew, W., \& Barnes, A. C. (2007). Streptococcus iniae: An aquatic pathogen of global veterinary significance and a challenging candidate for reliable vaccination. Veterinary Microbiology, 122(1-2), 1-15. https://doi.org/10.1016/j.vetmic.2007.03.002
Aksit, D. (2016). Balık yetiştiriciliğinde antibakteriyel direnç ve önemi. Turkiye Klinikleri Veterinary SciencesPharmacology and Toxicology-Special Topics, 2(1), 4754. https://www.turkiyeklinikleri.com/article/tr-balikyetistiriciliginde-antibakteriyel-direnc-ve-onemi75638.htm

Akyüz, A. P., Dernekbaşı, S., \& Karayücel, ì. (2018). Yüksek sıcaklıkla oksidatif strese maruz bırakılan gökkuşağı alabalı̆ında (Oncorhynchus mykiss Walbaum, 1792), karayemiş yaprağı (Laurocerasus officinalis Roem.) ekstraktının büyüme, yaşama oranı ve bazı antioksidan enzimler üzerine etkisi. Su Ürünleri Dergisi, 35(2): 131139. https://doi.org/10.12714/egejfas.2018.35.2.05

Almabrok, A. A., Amhamed, I. D., Mohamed, G. A., Bilen, S., \& Altief, T. A. S. (2018). Effect of Tilia tomentosa methanolic extract on growth performance, digestive enzyme activity, immune system and haematological indices of common carp (Cyprinus carpio). Marine Science and Technology Bulletin, 7(1), 12-20. https://doi.org/10.33714/masteb.421047

Altunoglu, Y. C., Bilen, S., Ulu, F., \& Biswas, G. (2017). Immune responses to methanolic extract of black cumin (Nigella sativa) in rainbow trout (Oncorhynchus mykiss). Fish \& Shellfish Immunology, 67, 103-109. https://doi.org/10.1016/j.fsi.2017.06.002

Amhamed, I. D., Mohamed, G. A., Almabrok, A. A., Altief, T. A. S., \& Bilen, S. (2018). Efficacy of dietary Chenopodium album extract on some health parameters, digestive enzymes and growth performance in juvenile Cyprinus carpio. Alınteri Zirai Bilimler Dergisi, 33(2), 165-176. https://doi.org/10.28955/alinterizbd.412455

Arslan, G., Sonmez, A. Y., \& Yanik, T. (2018). Effects of grape Vitis vinifera seed oil supplementation on growth, survival, fatty acid profiles, antioxidant contents and blood parameters in rainbow trout (Oncorhynchus mykiss). Aquaculture Research, 49(6), 2256-2266. https://doi.org/10.1111/are.13686

Austin, B., \& Austin, D.A. (2016). Aeromonadaceae representatives (motile aeromonads). In B. Austin \& D.A. Austin (Eds.), Bacterial fish pathogens: Disease of farmed and wild fish (sixth ed., pp 161-214). Springer. https://doi.org/10.1007/978-3-319-32674-0

Baba, E., Acar, U., Ontas, C., Kesbic, O. S., \& Yilmaz, S. (2016a). The use of Avena sativa extract against Aeromonas hydrophila and its effect on growth performance, hematological and immunological parameters in common carp (Cyprinus carpio). Italian Journal of Animal Science, 15(2), 325-333. https://doi.org/10.1080/1828051X.2016.1185977

Baba, E., Acar, U., Ontas, C., Kesbic, O. S., \& Yilmaz, S. (2016b). Evaluation of Citrus limon peels essential oil on growth performance, immune response of Mozambique tilapia Oreochromis mossambicus challenged with Edwardsiella tarda. Aquaculture, 465, 13-18. https://doi.org/10.1016/j.aquaculture.2016.08.023

Baba, E., Acar, U., Yilmaz, S., Ontas, C., \& Kesbic, O.S. (2017). Pre-challenge and post-challenge haematoimmunological changes in Oreochromis niloticus (Linnaeus, 1758) fed argan oil against Lactococcus garvieae. Aquaculture Research, 48(8), 4563-4572. https://doi.org/10.1111/are.13282

Baba, E., Acar, U., Yilmaz, S., Zemheri, F., \& Ergun, S. (2018). Dietary olive leaf (Olea europea L.) extract alters some immune gene expression levels and disease resistance to Yersinia ruckeri infection in rainbow trout Oncorhynchus 
mykiss. Fish \& Shellfish Immunology, 79, 28-33. https://doi.org/10.1016/j.fsi.2018.04.063

Baba, E., Ulukoy, G., \& Ontas, C. (2015). Effects of feed supplemented with Lentinula edodes mushroom extract on the immune response of rainbow trout, Oncorhynchus mykiss, and disease resistance against Lactococcus garvieae. Aquaculture, 448, 476-482. https://doi.org/10.1016/j.aquaculture.2015.04.031

Bilen, S., Altief, T. A. S., Ozdemir, K. Y., Salem, M. A. O., Terzi, E., \& Guney, K. (2020a). Effect of lemon balm (Melissa officinalis) extract on growth performance, digestive and antioxidant enzyme activities, and immune responses in rainbow trout (Oncorhynchus mykiss). Fish Physiology and Biochemistry, 46(1), 471-481.

https://doi.org/10.1007/s10695-019-00737-z

Bilen, S., Altunoglu, Y. C., Ulu, F., \& Biswas, G. (2016a). Innate immune and growth promoting responses to caper (Capparis spinosa) extract in rainbow trout (Oncorhynchus mykiss). Fish \& Shellfish Immunology, 57, 206-212. https://doi.org/10.1016/j.fsi.2016.08.040

Bilen, S., Karga, M., Altunoglu, Y. C., Ulu, F., \& Biswas, G. (2020b). Immune responses and growth performance of the aqueous methanolic extract of Malva sylvestris in Oncorhynchus mykiss. Marine Science and Technology Bulletin, 9(2), 159-167. https://doi.org/10.33714/masteb.746951

Bilen, S., Kenanoglu, O. N., Terzi, E., Ozdemir, R. C., \& Sonmez, A. Y. (2019). Effects of tetra (Cotinus coggygria) and common mallow (Malva sylvestris) plant extracts on growth performance and immune response in Gilthead sea bream (Sparus aurata) and European sea bass (Dicentrarchus labrax). Aquaculture, 734251. https://doi.org/10.1016/j.aquaculture.2019.734251

Bilen, S., Ozkan, O., Alagoz, K., \& Ozdemir, K. Y. (2018). Effect of dill (Anethum graveolens) and garden cress (Lepidium sativum) dietary supplementation on growth performance, digestive enzyme activities and immune responses of juvenile common carp (Cyprinus carpio). Aquaculture, 495, 611-616. https://doi.org/10.1016/j.aquaculture.2018.06.037

Bilen, S., Unal, S., \& Guvensoy, H. (2016b). Effects of oyster mushroom (Pleurotus ostreatus) and nettle (Urtica dioica) methanolic extracts on immune responses and resistance to Aeromonas hydrophila in rainbow trout (Oncorhynchus mykiss). Aquaculture, 454, 90-94. https://doi.org/10.1016/j.aquaculture.2015.12.010

Bilen, S., Yilmaz, S., \& Bilen, A. M. (2013). Influence of tetra (Cotinus coggygria) extract against Vibrio anguillarum infection in koi carp, Cyprinus carpio with reference to haematological and immunological changes. Turkish Journal of Fisheries and Aquatic Sciences, 13(3), 517522. https://doi.org/10.4194/1303-2712-v13_3_16

Capkin, E., Ozdemir, S., Ozturk, R. C., \& Altinok, I. (2017). Determination and transferability of plasmid-mediated antibiotic resistance genes of the bacteria isolated from rainbow trout. Aquaculture Research, 48(11), 55615575. https://doi.org/10.1111/are.13378

Capkin, E., Terzi, E., \& Altinok, I. (2015). Occurrence of antibiotic resistance genes in culturable bacteria isolated from Turkish trout farms and their local aquatic environment. Diseases of Aquatic Organisms, 114(2), 127-137. https://doi.org/10.3354/dao02852

Collins, M., Farrow, J., Phillips, B., \& Kandler, O. (1983). Streptococcus garvieae sp. nov. and Streptococcus plantarum sp. nov. Microbiology, 129(11), 3427-3431. https://doi.org/10.1099/00221287-129-11-3427

Cortez, R., Luna-Vital, D. A., Margulis, D., \& De Mejia, E. G. (2017). Natural pigments: stabilization methods of anthocyanins for food applications. Comprehensive Reviews in Food Science and Food Safety, 16(1), 180198. https://doi.org/10.1111/1541-4337.12244

Diler, O., \& Gormez, O. (2019). Gökkuşağı alabalıklarında (Oncorhynchus mykiss) bitkisel yem katkı maddelerinden Artemisia campestris L. ve Artemisia absinthium L'un bağırsak histomorfolojisi üzerine etkisi. Süleyman Demirel Üniversitesi Eğirdir Su Ürünleri Fakültesi Dergisi, 15(1), 10-18. https://doi.org/10.22392/egirdir.422547

Diler, O., Atabay, A., \& Gormez, O. (2017a). Bitkisel katkı maddesi Artemisia vulgaris' in gökkuşağı alabalıklarında (Oncorhyncus mykiss, Walbaum) büyüme performansı ve antioksidan aktivite üzerine etkisi. Süleyman Demirel Üniversitesi Eğirdir Su Ürünleri Fakültesi Dergisi, 13(2), 119-131.

https://dergipark.org.tr/tr/pub/egirdir/issue/30873/26 8454

Diler, O., Gormez, O., Diler, I., \& Metin, S. (2017b). Effect of oregano (Origanum onites L.) essential oil on growth, lysozyme and antioxidant activity and resistance against Lactococcus garvieae in rainbow trout, Oncorhynchus mykiss (Walbaum). Aquaculture Nutrition, 23(4), 844851. https://doi.org/10.1111/anu.12451

Diler, O., Gormez, O., Metin, S., Ilhan, I., \& Diler, I. (2017c). Origanum vulgare L. uçucu yağının gökkuşağı alabalıkları (Oncorhyncus mykiss)'nda büyüme, lizozim ve antioksidan aktivite ve Vibrio anguillarum'a karşı direnç üzerine etkisi. Süleyman Demirel Üniversitesi Eğirdir Su Ürünleri Fakültesi Dergisi, 13(1), 42-57. https://doi.org/10.22392/egirdir.252029

Diler, O., Gormez, O., Terzioglu, S., \& Atabay, A. (2018a). Pelin otu (Artemisia Vulgaris L)'nun gökkuşağı alabalıklarında (Oncorhynchus mykiss, walbaum) hastalıklara karşı direnç ve spesifik olmayan bağışıklık sistemi üzerine etkisi. Journal of Aquaculture Engineering and Fisheries Research, 4(1), 1-11. https://doi.org/10.3153/JAEFR18001

Diler, O., Gormez, O., Terzioglu, S., \& Bayrak, H. (2018b). Gökkuşağı alabalığı (Oncorhynchus mykiss) yetiştiriciliğinde görülen spironucleosis enfeksiyonlarının tedavisinde Artemisia campestris (L)'in kullanımı. Süleyman Demirel Üniversitesi Eğirdir Su Ürünleri Fakültesi Dergisi, 14(4), 312-323. https://doi.org/10.22392/egirdir.407905

Dugenci, S. K., \& Candan, A. (2001). Gökkuşağı alabalıklarında (Oncorhynchus mykiss, Walbaum) baz immunostimulanların spesifik olmayan bağışıklık sistemi üzerine etkisi. Turkısh Journal of Veterinary and Animal Sciences, 27(6), 1253-1260.

https://app.trdizin.gov.tr/publication/paper/detail/TXp BMk16ZzQ

Dugenci, S. K., Arda, N. \& Candan, A. (2003). Some medicinal plants as immunostimulant for fish. Journal of Ethnopharmacology, 88(1), 99-106. https://doi.org/10.1016/s0378-8741(03)00182-x

Duman, S., \& Sahan, A. (2018). Some hematological and nonspecific immune responses of rosehip (Rosa canina)-Fed Russian Sturgeon (Acipenser gueldenstaedtii Brandt \& Ratzeburg, 1833) to Mycobacterium salmoniphilum. Brazilian Archives of Biology and Technology, 61, 1-17. http://dx.doi.org/10.1590/1678-4324-2018180283 
Eldar, A., \& Ghittino, C. (1999). Lactococcus garvieae and Streptococcus iniae infections in rainbow trout Oncorhynchus mykiss: similar, but different diseases. Diseases of Aquatic Organisms, 36(3), 227-31. http://dx.doi.org/10.3354/dao036227

Er., A., \& Kayıs, S. (2015). Çay bitkisi (Camellia sinensis) tohumunun gökkuşağı alabalıklarında (Oncorhynchus mykiss) Aeromonas hydrophila enfeksiyonuna karşı kullanımının araştırılması. El-Cezeri Science and Engineering Journal, 2(3), 67-74. https://doi.org/10.31202/ecjse.67145

Ewing, W., McWhorter, A., Escobar, M., \& Lubin, A. (1965). Edwardsiella, a new genus of Enterobacteriaceae based on a new species, E. tarda. International Bulletin of Bacteriological Nomenclature and Taxonomy, 15(1), 3338. https://doi.org/10.1099/00207713-15-1-33

FAO (2018). FAO Yearbook, Fishery and Aquaculture Statistics. http://www.fao.org/3/cb1213t/CB1213T.pdf

Frans, I., Michiels, C. W., Bossier, P., Willems, K. A., Lievens, B. \& Rediers, H. (2011). Vibrio anguillarum as a fish pathogen: Virulence factors, diagnosis and prevention. Journal of Fish Diseases, 34(9), 643-661. https://doi.org/10.1111/j.1365-2761.2011.01279.x

Gauthier, D. T. (2015). Bacterial zoonoses of fishes: A review and appraisal of evidence for linkages between fish and human infections. The Veterinary Journal, 203(1), 27-35. https://doi.org/10.1016/j.tvjl.2014.10.028

Gudding, R., Lillehaug, A., \& Evensen, O. (2014). Fish Vaccination. John Wiley \& Sons. https://doi.org/10.1002/9781118806913

Gulec, A. K., Danabas, D., Ural, M., Seker, E., Arslan, A., \& Serdar, O. (2013). Effect of mixed use of thyme and fennel oils on biochemical properties and electrolytes in rainbow trout as a response to Yersinia ruckeri infection. Acta Veterinaria Brno, 82(3), 297-302. https://doi.org/10.2754/avb201382030297

Gullu, K., Acar, U., Kesbic, O. S., Yilmaz, S., Agdamar, S., Ergun, S., \& Turker, A. (2016). Beneficial effects of oral allspice, Pimenta dioica powder supplementation on the hematoimmunological and serum biochemical responses of Oreochromis mossambicus. Aquaculture Research, 47(9), 2697-2704. https://doi.org/10.1111/are.12717

Gultepe, N. (2020). Protective effect of d-limonene derived from orange peel essential oil against Yersinia ruckeri in rainbow trout. Aquaculture Reports, 18, 100417. https://doi.org/10.1016/j.aqrep.2020.100417

Gultepe, N., Acar, U., Kesbic, O. S., Yilmaz, S., Yildirim, O., \& Turker, A. (2014). Effects of dietary Tribulus terrestris extract supplementation on growth, feed utilization, hematological, immunological, and biochemical variables of Nile tilapia Oreochromis niloticus. The Israeli Journal of Aquaculture-Bamidgeh, 66, 1-9. https://doi.org/10.46989/001c.20774

Guven, A., \& Yalcin, S. (2017). Effects of dietary yeast autolysate on performance, some blood parameters and lysozyme activity in rainbow trout (Oncorhynchus mykiss). Ankara Üniversitesi Veteriner Fakültesi Dergisi, 64(3), 177-182. https://doi.org/10.1501/Vetfak_0000002796

Horne, M. T., \& Barnes, A. C. (1999). Enteric redmouth disease (Yersinia ruckeri), in P.T.K. Woo and D.W. Bruno (Eds.), Fish Diseases and Disorders, Viral, Bacterial and Fungal Infections, CABI Publishing, Wallingford.

Ispir, U., \& Dorucu, M. (2005). A study on the effects of levamisole on the immune system of rainbow trout
(Oncorhynchus mykiss, walbaum). Turkish Journal of Veterinary and Animal Sciences, 29, 1169-1176. https://dergipark.org.tr/tr/download/articlefile/132674

Kesbic, O. S. (2019a). Effects of juniper berry oil on growth performance and blood parameters in common carp (Cyprinus carpio). Aquaculture Research, 50(1), 342-349. https://doi.org/10.1111/are.13908

Kesbic, O. S. (2019b). Effects of the cinnamon oil (Cinnamomum verum) on growth performance and blood parameters of rainbow trout (Oncorhynchus mykiss). Turkish Journal of Agriculture-Food Science and Technology, 7(2), 370-376. https://doi.org/10.24925/turjaf.v7i2.370-376.2360

Kesbic, O. S., Acar, U., Yilmaz, S., \& Aydin, O. D. (2020a). Effects of bergamot (Citrus bergamia) peel oil-supplemented diets on growth performance, haematology and serum biochemical parameters of Nile tilapia (Oreochromis niloticus). Fish Physiology Biochemistry, 46, 103-110. https://doi.org/10.1007/s10695-019-00700-y

Kesbic, O. S., Parrino, V., Acar, U., Yilmaz, S., Paro, G. L., \& Fazio, F. (2020b). Effects of Monterey cypress (Cupressus macrocarpa Hartw) leaf essential oil as a dietary supplement on growth performance and haematological and biochemical parameters of common carp (Cyprinus carpio L.). Annals of Animal Science, 1-33. https://doi.org/10.2478/aoas-2020-0041

Kıvrak, E \& Didinen, B.I. (2017). Gökkuşağı alabalığı (Oncorhynchus mykiss, Walbaum 1792)'nın yemlerine biberiye bitki (Rosmarinus officinalis) yağı ilavesinin balıkların büyüme performansı ve bazı kan parametrelerine olan etkileri. Yunus Research Bulletin, 2 193-202. https://doi.org/10.17693/yunusae.vi.287094

Lee, C. S. (2015). Dietary Nutrients, Additives and Fish Health. Wiley-Blackwell. https://doi.org/10.1002/9781119005568

Ozturk, R. C., \& Altinok, I. (2014). Bacterial and viral fish diseases in Turkey. Turkish Journal of Fisheries and Aquatic Sciences, 14(1), 275-297. https://doi.org/10.4194/1303-2712-v14_1_30

Parrino, V., Kesbic, O. S., Acar, U., \& Fazio, F. (2019). Hot pepper (Capsicum sp.) oil and its effects on growth performance and blood parameters in rainbow trout (Oncorhynchus mykiss). Natural Product Research, 34(22), 3226-3230 https://doi.org/10.1080/14786419.2018.1550769

Öntaş, C., Uluköy, G., BABA, E., \& Mammadov, R. (2020). Crocus cancellatus subsp. mazziaricus (Herbert) mathew bitki ekstraktının avrupa deniz levrek balığı (Dicentrarchus labrax, L. 1758) doğal bağışıklık sistemi üzerine etkisi. Acta Aquatica Turcica, 16(1), 148-157. https://doi.org/10.22392/actaquatr.622606

Ravelo, C., Magarinos, B., López-Romalde, S., Toranzo, A. E., \& Romalde, J. L. (2003). Molecular fingerprinting of fishpathogenic Lactococcus garvieae strains by random amplified polymorphic DNA analysis. Journal of Clinical Microbiology, 41(2), 751-756. https://doi.org/10.1128/JCM.41.2.751-756.2003

Rehulka, J., Marejková, M., \& Petráš, P. (2012) Edwardsiellosis in farmed rainbow trout (Oncorhynchus mykiss). Aquaculture Research, 43(11), 1628-1634. https://doi.org/10.1111/j.1365-2109.2011.02968.x

Sahan, A., \& Duman, S. (2010). Influence of \beta-1, 3/1, 6 glucan applications on some non-specific cellular immune response and haematologic parameters of 
healthy Nile tilapia (Oreochromis niloticus L., 1758). Turkish Journal of Veterinary and Animal Sciences, 34(1), 75-81. https://doi.org/10.3906/vet-0810-21

Sahan, A., Duman, S., Colak, S. O., Cinar, E., \& Bilgin, R. (2017). Determination of some hematological and non-specific immune defences, oxidative stress and histopathological status in rainbow trout (Oncorhynchus mykiss) fed rosehip (Rosa canina) to Yersinia ruckeri. Turkish Journal of Fisheries and Aquatic Sciences, 17(1), 91-100. https://doi.org/10.4194/1303-2712-v17_1_11

Sahan, A., Ozutok, S., \& Kurutas, E.B. (2016). Determination of some hematological parameters and antioxidant capacity in Nile tilapia (Oreochromis niloticus Linnaeus, 1758) fed ginger (Zingiber Officinale Roscoe) to Aeromonas hydrophila. Turkish Journal of Fisheries and Aquatic Sciences, 16, 197-204. https://doi.org/10.4194/1303-2712-v16_1_20

Savaşer, S., Akçimen, U., Ceylan, M., Bektaş, Z.H., Yener, O., \& Bulut, C. (2019). Zencefil (Zingiber officinale)'in gökkuşağı alabalıklarında (Oncorhynchus mykiss Walbaum) immunostimulant ve büyüme destekleyici olarak kullanımı. Journal of Limnology and Freshwater Fisheries Research 5(2), 121-135. https://doi.org/10.17216/limnofish.487812

Schleifer, K. H., Klaus, J., Dvorak, C., Kilpper-Balz, R., Collins, M. D., \& Fischer, W. (1985). Transfer of Streptococcus lactis and related streptococci to the genus Lactococcus gen. nov. Systematic and Applied Microbiology, 6(2), 183195. https://doi.org/10.1016/S0723-2020(85)80052-7

Teixeira, L. M., Merquior, V. L. C., Vianni, C. E., Carvalho, G. S., Fracalanzza, S. E. L., Steigerwalt, A. G., Brenner, D. J., \& Facklam, R. R. (1996). Phenotypic and genotypic characterization of a typical Lactococcus garvieae strains isolated from water buffalos with subclinical mastitis and confirmation of L. garvieae as a senior subjective synonym of Enterococcus seriolicida. International Journal of Systematic and Evolutionary Microbiology, 46(3), 664-668. https://doi.org/10.1099/00207713-463-664

Terzioglu, S., \& Diler, O. (2016). Effect of dietary sage (Salvia officinalis L.), licorice root (Glycyrrhize glabra L.), blueberry (Vaccinium myrtillus L.) and echinaceae (Echinacea angustifolia Hell) on nonspecific immunity and resistance to Vibrio anguillarum infection in rainbow trout, (Oncorhynchus mykiss). Süleyman Demirel Üniversitesi Eğirdir Su Ürünleri Fakültesi Dergisi, 12(2), 110-118. https://doi.org/10.22392/egirdir.284921

TUIK (2019). Su ürünleri istatistikleri. https://data.tuik.gov.tr/Kategori/GetKategori?p=tarim$111 \&$ dil $=1$

Ulukoy, G., Baba, E., \& Birincioglu, S. S. (2016). Effects of dietary Lentinula edodes extract on liver and gut histology of rainbow trout (Oncorhynchus mykiss, Walbaum 1792). Fish \& Shellfish Immunology, 53, 106. https://doi.org/10.1016/j.fsi.2016.04.068

Ulukoy, G., Metin, S., Kubilay, A., Guney, S., Yildirim, P., GuzelSeydim, Z., Kok-Tas, T., \& Gumus, E. (2017). The effect of kefir as a dietary supplement on nonspecific immune response and disease resistance in juvenile rainbow trout, Oncorhynchus mykiss (Walbaum 1792). Journal of the World Aquaculture Society, 48(2), 248-256. https://doi.org/10.1111/jwas.12336

Woo, P.T.K., \& Bruno, D.W. (2017). Fish diseases and disorders:, Volume 3: Viral, bacterial and fungal infections., In: Woo PTK, Cipriano RC (eds): Wallingford, UK, CAB International.

Woo, P. T. K., \& Cipriano, R. C. (2017). Fish viruses and bacteria: pathobiology and protection. CABI Publishing. Wallingford, UK. https://doi.org/10.1079/9781780647784.0000

Yarsan, E. (2013). Veteriner hekimlikte antibiyotikler (pratik bilgiler rehberi). Güneş Tıp Kitabevi.

Yildirim, O. (2008). Aquafeed industry in Turkey: its aquafeed projections towards the year 2015. Turkish Journal of Fisheries and Aquatic Sciences, 8(1), 93-98. https://www.trjfas.org/abstract.php?lang=en\&id=595

Yilmaz, S., Ergun, S., Kaya, H., \& Gurkan, M. (2014). Influence of Tribulus terrestris extract on the survival and histopathology of Oreochromis mossambicus (Peters, 1852) fry before and after Streptococcus iniae infection. Journal of Applied Ichthyology, 30(5), 994-1000. https://doi.org/10.1111/jai.12458

Yilmaz, E. (2019d). Effects of dietary anthocyanin on innate immune parameters, gene expression responses, and ammonia resistance of Nile tilapia (Oreochromis niloticus). Fish \& Shellfish Immunology, 93, 694-701. https://doi.org/10.1016/j.fsi.2019.08.033

Yilmaz, E. (2020). Effect of dietary carob (Ceratonia siliqua) syrup on blood parameters, gene expression responses and ammonia resistance in tilapia (Oreochromis niloticus). Aquaculture Research, 51(5), 1903-1912. https://doi.org/10.1111/are.14540

Yilmaz, E., \& Er, M. (2019). Effects of figs and rosemary extracts on rainbow trout (Oncorhynchus mykiss) on growth performance and blood parameters. Acta Aquatica Turcica, 15(1), 19-25. https://doi.org/10.22392/egirdir.429630

Yilmaz, E., Celik, E. S., Ergun., S., \& Yilmaz, S. (2020b). Effects of dietary olive pomace oil on growth performance, some Immune parameters and disease resistance (Lactococcus garvieae) of rainbow trout (Oncorhynchus mykiss). Journal of Anatolian Environmental and Animal Sciences, 5(4), 597-604. https://doi.org/10.35229/jaes.798086

Yilmaz, E., Ergun, S., \& Yilmaz S. (2020a). Effects of supplementation of L-alliin and oleuropein into rainbow trout (Oncorhynchus mykiss) feeds on growth performance, some immune parameters and disease resistance (Aeromonas salmonicida subsp. salmonicida). Journal of Limnology and Freshwater Fisheries Research, 6(3), 180-188.

https://doi.org/10.17216/limnofish.746677

Yilmaz, E., Ergun, S., \& Yilmaz, S. (2015b). Influence of carvacrol on the growth performance, hematological, non-specific immune and serum biochemistry parameters in rainbow trout (Oncorhynchus mykiss). Food and Nutrition Sciences, 6(5), 523-531. https://doi.org/10.4236/fns.2015.65054

Yilmaz, S. (2019a). Effects of dietary caffeic acid supplement on antioxidant, immunological and liver gene expression responses, and resistance of Nile tilapia, Oreochromis niloticus to Aeromonas veronii. Fish \& Shellfish Immunology, 86, 384-392. https://doi.org/10.1016/j.fsi.2018.11.068

Yilmaz, S. (2019b). Karabaş otu (Lavandula stoechas) yağının sazan balığı (Cyprinus carpio) yemlerine ilavesinin büyüme performansı ve bazı kan parametreleri üzerine etkileri. ÇOMÜ Ziraat Fakültesi Dergisi, 7(1), 187-193. https://doi.org/10.33202/comuagri.451304 
Yilmaz, S. (2019c). Effects of dietary blackberry syrup supplement on growth performance, antioxidant, and immunological responses, and resistance of Nile tilapia, Oreochromis niloticus to Plesiomonas shigelloides. Fish \& Shellfish Immunology, 84, 1125-1133. https://doi.org/10.1016/j.fsi.2018.11.012

Yilmaz, S., \& Ergun, S. (2014). Dietary supplementation with allspice Pimenta dioica reduces the occurrence of streptococcal disease during first feeding of Mozambique tilapia fry. Journal of Aquatic Animal Health, 26(3), 144-148. https://doi.org/10.1080/08997659.2014.893459

Yilmaz, S., \& Ergun, S. (2018). Trans-cinnamic acid application for rainbow trout (Oncorhynchus mykiss): I. Effects on haematological, serum biochemical, non-specific immune and head kidney gene expression responses. Fish \& Shellfish Immunology, 78, 140-157. https://doi.org/10.1016/j.fsi.2018.04.034

Yilmaz, S., Acar, U., Kesbic, O. S., Gultepe, N., \& Ergun, S. (2015a). Effects of dietary allspice, Pimenta dioica powder on physiological responses of Oreochromis mossambicus under low pH stress. SpringerPlus, 4(1), 719. https://doi.org/10.1186/s40064-015-1520-7

Yilmaz, S., Ergun, S., \& Celik, E. S. (2013a). Effect of dietary herbal supplements on some physiological conditions of sea bass Dicentrarchus labrax. Journal of Aquatic Animal Health, 25(2), 98-103. https://doi:10.1080/08997659.2013.768561

Yilmaz, S., Ergun, S., \& Celik, E. S. (2018b). The effect of dietary carob (Ceratonia siliqua) syrup on growth performance, haematological, serum biochemical and immunological parameters in tilapia (Oreochromis mossambicus). Turkish Journal of Agriculture - Food Science and Technology, 6(12), 1820-1826. https://doi.org/10.24925/turjaf.v6i12.1820-1826.2184

Yilmaz, S., Ergun, S., \& Celik, E.S. (2016). Effect of dietary spice supplementations on welfare status of sea bass, Dicentrarchus labrax L. Proceedings of the National Academy of Sciences, India Section B: Biological Sciences, 86(1), 229-237. https://doi.org/10.1007/s40011-014-0444-2

Yilmaz, S., Ergun, S., \& Soytas, N. (2013b). Herbal supplements are useful for preventing streptococcal disease during first-feeding of tilapia fry, Oreochromis mossambicus. The Israeli Journal of Aquaculture-Bamidgeh, 65, 1-5. https://ija.scholasticahq.com/issue/2837
Yilmaz, S., Ergun, S., \& Soytas, N. (2013c). Dietary supplementation of cumin (Cuminum cyminum) preventing streptococcal disease during first-feeding of Mozambique tilapia (Oreochromis mossambicus). Journal of BioScience and Biotechnology, 2(2), 117-124. http://www.jbb.uni-

plovdiv.bg/documents/27807/59545/jbb_20132\%282\%29-pages_117-124.pdf/57e419a9-12f8-46ee9a46-8daa97447acd?version $=1.0$

Yilmaz, S., Ergun, S., \& Turk, N. (2012). Effects of cuminsupplemented diets on growth and disease (Streptococcus iniae) resistance of tilapia (Oreochromis mossambicus). The Israeli Journal of AquacultureBamidgeh, 64, 1-5. http://hdl.handle.net/10524/23593

Yilmaz, S., Ergun, S., Celik, E. S., Yigit, M., \& Bayizit, C. (2019a). Dietary trans-cinnamic acid application for rainbow trout (Oncorhynchus mykiss): II. Effect on antioxidant status, digestive enzyme, blood biochemistry and liver antioxidant gene expression responses. Aquaculture Nutrition, 25(6), 1207-1217. https://doi.org/10.1111/anu.12935

Yilmaz, S., Ergun, S., Yigit, M., Yilmaz, E., \& Ahmadifar, E. (2020c). Dietary supplementation of black mulberry (Morus nigra) syrup improves the growth performance, innate immune response, antioxidant status, gene expression responses, and disease resistance of Nile tilapia (Oreochromis niloticus). Fish \& Shellfish Immunology, 107, 211-217. https://doi.org/10.1016/j.fsi.2020.09.041

Yilmaz, S., Sova, M., \& Ergun, S. (2018a). Antimicrobial activity of trans-cinnamic acid and commonly used antibiotics against important fish pathogens and nonpathogenic isolates. Journal of Applied Microbiology, 125(6), 17141727. https://doi.org/10.1111/jam.14097

Yonar, M. E., Yonar, S. M., Ispir, U., \& Ural, M. S. (2019). Effects of curcumin on haematological values, immunity, antioxidant status and resistance of rainbow trout (Oncorhynchus mykiss) against Aeromonas salmonicida subsp. achromogenes. Fish \& Shellfish Immunology, 89, 83-90. https://doi.org/10.1016/j.fsi.2019.03.038

emheri-Navruz, F., Acar, U., \& Yilmaz, S. (2019). Dietary supplementation of olive leaf extract increases haematological, serum biochemical parameters and immune related genes expression level in common carp (Cyprinus carpio) juveniles. Fish \& Shellfish Immunology, 89, 672-676. https://doi.org/10.1016/j.fsi.2019.04.037 